some length in more than one institution. I believe, however, that "sufficient evidence is seldom available at the time of referral to justify an individual psychiatrist recommending detention of a patient." 1 In addition, I would quote Courtney Wade's perceptive comment: "The psychiatrist, however, can treat the patient only on the findings and the history. Where the history is a fabrication, then the findings and treatment are based on false premises."'2

A schizophrenic once challenged me with the following couplet:

You want to get inside my head and see the world within;

But can you get inside my head and see the world without?

That, Sir, is the sixty-four thousand dollar question. I fear the answer is "No."

ANDREW BAMJI

Middlesex Hospital,

'Blackwell P. Guy's Hospital Reports 1965;114: 2 Wade CC. Med Care 1965;3:189-92.

${ }_{*}^{*}$ This correspondence is now closed.-ED, $B M \mathcal{B}$.

\section{Treatment of sciatica}

SIR,-In reply to Dr Michael Snaith's letter (1 November, $p$ 1217), what is classical sciatica? I was taught that sciatica is a pain in the lower limb in the distribution of the sciatic nerve. This contains elements from the nerve roots of L4, L5, S1, and S2. The first three nerve roots can be compressed by a lumbar intervertebral disc, but they also produce the nerve supply to ligaments in the lower back and the sacroiliac joints and these ligaments, when strained, can refer pain to the dermatome of their nerve supply. It is a rarity for the S2 nerve root to be hit by a disc, but pain in the S2 dermatome-namely, at the back of the thigh and behind the knee-is a fairly common occurrence, this being caused by a strain of the sacrotuberous ligament. No investigation can prove a ligamentous cause of sciatica.

By my interpretation of the symptoms and signs on examination, the lumbar disc causes low back pain and sciatica in less than $50 \%$ of cases and only $1 \%$ require surgery.

London W1

RONALD BARBOR

\section{Hidden hypotheses}

SIR,-I have a great respect for both Professor J D Swales and Dr Mildred Seelig, and therefore read his distinguished review of her book on magnesium metabolism with much interest (25 October, $p$ 1132). The review itself was perfectly fair, but why has it become so fashionable for professors of medicine of positivist cast of mind to use any opportunity and the authority of Karl Popper to take a side swipe at psychoanalysis even when writing about mineral metabolism, or some other topic in their own field?

It would be my view that psychology found its Newton in Freud; but it took physics nearly 300 years to travel via Kant to Einstein and on the way it dallied for long periods with the kind of all-embracing explanatory concepts such as phlogiston and the ether that Professo Swales finds unacceptable in psychoanalysis and which may in fact have nourished rather than retarded its growth as a science. We do not find scientists such as the late Lord Adrian, working in fields closer to those tilled by psychoanalysts than Professor Swales's, sneering at their work, which like that of modern physicists needs to be relativistic and is subject to the uncertainty principle because the presence of the observer impinges so greatly on what is observed. Sooner or later psychology will find what Kant calls the true path of science and meanwhile it is properly concerned with the content as well as the forms of the mental processes of those suffering from psychiatric disorders-which are, like other diseases, experiments of nature able to throw light on normal function.

It was Freud himself who said that credulity was a necessary quality for the creative scientist and to be over-critical is, as Roy Campbell put it in relation to poetry, making bit and bridle more important than the horse. Perhaps seekers after the truth in any particular field of inquiry should like journeymen stick to their lasts; for, in the words of Hooker, labour involved in digging up in order to look at the philosophical roots of our assumptions is more necessary than pleasant.

University Department of Paediatrics,

JOHN DAvis Addenbrooke's Hospital

\section{Suppression of "rubral" tremor with levodopa}

SIR,-I have always considered that rubral tremor was present at rest and was exacerbated by movement when a cerebellar component was obvious. It is distinguished from a Parkinsonian tremor by being much more a flexion-extension movement of the wrist and fingers than the side-to-side pill-rolling tremor of Parkinsonism. It is not associated usually with paucity of movement or with cogwheel rigidity. In five patients whom I have treated deliberately with Sinemet or Madopar the response has been completely disappointing, and I was interested to see the report by Drs L J Findley and M A Gresty (18 October $p$ 1043) of a case in which levodopa seemed of some value in such a tremor. Perhaps the different response to drugs is due to an underlying variation of pathophysiology that produces the similar clinical picture.

Department of Neurology,

North Manchester General Hospital,
Manchester M8 6RB

\section{Electricity and bones}

SIR,-The 16 August (p 470) issue of $B M F$ has just come to my attention, and I was somewhat surprised to learn that "electrical treatment will, in time, become a useful addition to the orthopaedic armamentarium." This statement appears along with others equally perplexing in a leading article entitled "Electricity and bones." Apparently, you have not kept completely up to date with the latest developments in this rapidly expanding field. Furthermore, you make a mistake common to many neophytes in this new discipline of electrobiology.

Firstly, it is inappropriate to consider repair of fresh fractures and ununited fractures in common biological terms. One is an example of an acute repair process in which injury potentials and other factors trigger the formation of callus de novo. In the non-union, repair tissues already exist and are relatively quiescent. All of the evidence from animals and patients with non-unions treated with pulsing electromagnetic fields points to an evolution in the soft tissues (fibrocartilage and fibrous tissue) occupying the gap between the bone ends. No new callus has been observed during nonunion healing with pulsing electromagnetic fields. In fact, the mechanism involves mineralisation of the fibrocartilage, vascular penetration, removal of the provisionally "calcified material," and its replacement by bone. The process resembles closely normal endochondral ossification. Furthermore, recent studies have demonstrated that pulse characteristics which are highly effective in treating non-unions in the clinic have little or no effect on fresh fractures in animals. This specificity is in consonance with other in vitro and animal studies which have demonstrated selective biological actions of different pulse waveforms.

In the second mistake, the author has made little or no biophysical distinction between constant currents delivered by electrodes and pulsing currents induced in tissues by time-varying electromagnetic fields. From both physical and biological viewpoints effects of the two different systems may be quite different. This tendency to lump all electrical modalities together can only stultify our intellectual growth curve. It is as if we said that red and green are the same because both are colours.

Finally, I should like to bring to the attention of your readers an alternative view to the one expressed by your editorial-namely, "electrical treatment is now a useful addition to the orthopaedic armamentarium." In the programme with pulsing electromagnetic fields, the worldwide experience involves more than 2000 orthopaedic surgeons who have treated more than 3500 patients with this modality. In the first 1000 end results, the overall success rate is $80 \%$ for all categories of ununited bone discontinuities, including nonunions, failed fusions, and congenital "pseudarthroses." For tibial non-unions in adults, the success rate approaches $90 \%$ and compares most favourably with the best series involving operative repair. In many of these patients, particularly those with active infections and congenital "pseudarthroses," amputation has been avoided when al else has failed. Here in the United States the method has been approved as safe and effective by the Food and Drug Administration, and is universally available to the orthopaedic community.

I am afraid that many of your readers will interpret the tone of the leading article in such a way that the vast benefits of this breakthrough in controlling cell function may be denied to those who could benefit from its use. It would seem to me from reports reaching our shores that your country is beset by at least as many financial pressures on its economy as ours. Even if the risk factors were equal, the cost of a surgical repair, as opposed to outpatient care with pulsing electromagnetic fields, does not make good economic sense here, and may not in England. This takes into account your National Health Service system with a relatively fixed number of hospital beds and services. If the bed is occupied by a patient with non-union awaiting surgery, it cannot be occupied by a patient with osteoarthritis awaiting a hip prosthesis, or for that matter a patient with an acute myocardial infarction.

\section{ANDREW L BASSETT}

Department of Orthopedic

Department

College of Physicians and

Surgeons of Columbia

University,
New York, NY 10032, USA

${ }^{*} *$ Professor Bassett suggests that no proper understanding or reference is made in our leading article to the different types of electrical 
stimulus which can be applied. This is quite incorrect, since in the sixth and seventh paragraphs we have set out the problem of the different types of stimulus which might be applied. The fact that we find it extremely difficult to satisfy ourselves that any one method is better than another, or that Professor Bassett's technique is immeasurably superior in any respect, is simply the result of the difficulty of making much sense out of much of the literature on this subject-which has failed, in our opinion, to show any clear relationship between the effectiveness of one type of stimulus and that of another. Certainly, some methods are more convenient than others but this is not the same thing. In America this method of treating fractures may well be already established. In Britain ou experience is still at an early stage and therefore the merits of the management of fractures by such methods must still be regarded as improved.

The economics are another question, which clearly we cannot discuss rationally until we are quite certain of the clinical effectiveness of the method. If we knew, for example, that we could guarantee a satisfactory outcome at least as quickly as by conventional methods of treating non-union, we could then begin to compare the costs of the relatively short period of time in hospital for the operation with the somewhat high costs of the currently available electrobiological apparatus. Of course we welcome clinical endeavours in assessing the place of these methods in Britain but we are entitled to make haste slowly.-ED, $B M \mathcal{~}$.

\section{Yaws again}

SIR,-Your leading article "Yaws again" (25 October, p 1090) is a timely reminder that yaws has reappeared on the tropical scene despite seemingly successful eradication programmes, but ignores the most obvious reason for this sad state of affairs. Three reasons are given for failure to eradicate the disease, but no mention is made of poverty and the poor living conditions associated with it: conditions in which yaws thrives-as, for that matter, do many other "tropical" diseases.

I suspect that doctors who approach the problem of eradicating yaws in strictly medical terms are those who consider that medicine and politics do not mix. Surely the lesson of yaws is that there are times when they must.

W H JOPLING

London SE25 6PN

\section{Anaphylactic reaction to desensitisation}

SIR,-Anaphylactic death is always a dramatic medical tragedy. The chest physician who suggested hyposensitisation for a patient with asthma and allergic rhinitis did so when sodium cromoglycate had failed to give adequate relief. The tyrosene-adsorbed house dust mite extract of Dermatophagoides pteronyssinus (Migen) caused anaphylactic death in a patient (27 September, p 854). Dr Pamela W Ewan (18 October, p 1069) states: "in the past (sic) hyposensitisation was widely used in the treatment of allergic disorders; however, impressions of benefit were often based on anecdote and where controlled trials have been performed results are variable." There is no doubt that in properly carried out controlled trials in pollen, cat, and mite allergic patients specific immunotherapy does give benefit.

Noon, working at St Mary's Hospital, reported the first successful treatment of seasonal hay fever by injection therapy in $1911 .^{1} \mathrm{His}$ work was based on unsound theory, but even in 1980 we do not know why hyposensitisation helps the allergic patient, but this should not be a reason for advising against injections. We therefore come back to the question of the danger of hyposensitisation. Tyrosine-adsorbed preparations such as Migen have a relatively short half life and may well be potentially dangerous, and deaths have occurred with its use. The alum pyridine preparation Allpyral, which has a world wide use, has a long half life. It became available in the UK in 1965. In this country over four million injections of this vaccine have been given and, according to the DHSS, no deaths have been reported from its use. It is not therefore correct to damn all hyposensitisation injections as dangerous.

London W1N 1DJ

A W FrankLaND

${ }^{1}$ Noon L. Lancet 1911 ; : 1572-3.

SIR,-We would take issue with the letter written by Dr Pamela Ewan (18 October, p 1069). The patient whose death from anaphylactic shock following hyposensitisation was reported by Dr David A Rands (27 September, $p$ 854), though she was being treated "predominantly for allergic rhinitis," was also being treated for her asthma. There are between 1000 and 2000 deaths per year in the UK from this condition and many of these occur unpredictably even in patients with apparently "mild disease" who may be well controlled on symptomatic therapy. This can be compared with only 13 reported death following anaphylactic reaction to hyposensitisation with Bencard vaccines since 1966 an estimated incidence of 1 in 750000 in terms of injections administered.

Recently carefully controlled trials of hyposensitisation treatment of asthma have shown substantial benefit in patients who are correctly selected, ${ }^{\mathbf{1}-3}$ and while certain controversy may still be voiced about efficacy many clinicians now accept the value of this form of treatment in appropriate cases.

It must be remembered that manipulation of immunological processes is not without its problems but general reactions are uncommon and fatal anaphylactic reactions, although tragic and regrettable, are nevertheless rare. We would endorse the sentiments of Dr Rands that doctors using hyposensitisation should have immediate access to the drugs necessary for treating anaphylactic reactions, which should be used promptly as specified in the detailed literature accompanying each dispensed course of Bencard allergy vaccine.

RAY ANCILI

GILLIAN WAINSCOTT

Medical Department,

Bencard,

' Aas K. Acta Paed Scand 1971;60:264.
2 D'Souza MF, Pepys J, Wells ID, et al. Clin Allergy 1973;3:177-93.

Warner JO, Price JF, Soothill JF, Hey EN. Lancet 1978 ;ii:912-5.

SIR,-I read with interest Dr D A Rand's paper on a patient who died after being given a desensitising vaccine (27 September, p 854). I recently had a similar case. At the end of July 1980 a 24-year-old male patient in my practice who had suffered from bronchial asthma for 16 years died of status asthmaticus after his 15th injection of SDV Bencard (a desensitising vaccine).

The onset of status asthmaticus did not occur until about 25 minutes after the injection -that is, outside the period of supervision recommended by the maker of the vaccine. Despite intense resuscitative measures the patient died one hour after the injection had been given. He had never previously suffered from status asthmaticus, and his asthma was considered of moderate severity only.

R C H Pollard

Plymouth, Devon

\section{New system for single-needle dialysis}

SIR,-We are pleased to note that Dr John Cunningham and others (25 October, p 1109) were able to reproduce similar results to us, although we achieved ours through a simpler, cheaper, and safer single-needle dialysis system. ${ }^{1}$ One of our home-dialysis patients has been using this system for five years. Currently we have 16 patients at home and nine patients on hospital dialysis using our single-needle system satisfactorily.

R AHMAD

B LARGE

D REDMAN

Renal Dialysis Unit,

Sefton Generai Hospital
Liverpool L15 $2 \mathrm{HE}$

'Ahmad R, Large B, Redman D. Dialysis Transplant $1979 ; 8: 260-3$

\section{Training requirements for cosmetic surgery}

SIR,-Over the last 10 years there has been an increase in public demand for cosmetic surgery. This has raised problems within the profession in that there is at present in this country no recognised training programme for this specialty. Surgeons entering the specialty do so after training in differing disciplines of surgery.

The British Association of Cosmetic Surgeons is most concerned that adequate standards are achieved and maintained in the specialty and have sought the co-operation of the Royal Colleges of Surgeons of England, Ireland, Edinburgh, and Glasgow in formulating minimal training requirements for this specialty. In this way it is hoped that cosmetic surgery will be recognised as a distinct specialty within the profession as it is already in North America and many European countries.

\section{C HERBERT Vice-president N G BuchaN Honorary secretary}

British Association of Cosmetic Surgeons, London W1

\section{Certification-continuing arguments}

SIR,-Mr Michael Lowe suggests in his article on certification (25 October, p 1155) that the regional medical service could employ GPs on a part-time basis to examine self- 